\title{
Comparação Entre a Análise Visual e a Computadorizada de Registros Cardiotocográficos Anteparto em Gestaçōes de Alto Risco.
}

Autor: Corintio Mariani Neto

Orientador: Prof. Dr. Anibal Faúndes

Tese apresentada à Faculdade de Ciências Médicas da Universidade Estadual de Campinas para obtenção do Título de Doutor em Medicina, na área de Tocoginecologia em 21/10/99.

A cardiotocografia anteparto tem sido amplamente utilizada nas últimas duas décadas para avaliação da vitalidade fetal em gestações de alto risco e, há algum tempo, vem recebendo críticas em relação à reprodutibilidade dos laudos emitidos por interpretação visual. Recentemente, introduziu-se a análise computadorizada a fim de eliminar as possiveis dúvidas do examinador na leitura dos registros. Com o objetivo de avaliar a consistência da análise visual de dois observadores independentes e compará-la à computadorizada, quanto ao laudo final e suas implicações clínicas, realizou-se este estudo em 120 gestantes atendidas no Hospital - Maternidade Leonor Mendes de Barros, em São Paulo. Avaliou-se o grau de concordância entre as análises visuais independentes dos dois observadores e entre o método da análise visual e da computadorizada (System 8002), bem como, compararam-se os indicadores de desempenho dos dois métodos em relação a parâmetros indicativos de resultado perinatal anormal: $\mathrm{pH}$ do sangue da artéria umbilical < 7,20; índice de Apgar de 1 e 5o minutos < 7; admissão na unidade de terapia intensiva neonatal e internação do recém-nascido por mais de 7 dias. Para análise estatística, foram utilizados o coeficiente kappa, o teste exato de Fisher, a distribuição binomial e o teste de McNemar para amostras emparelhadas. Os resultados mostraram boa taxa de concordância na interpretação dos observadores $(92,5 \%$; kappa $=0,76)$, enquanto que a comparação entre os dois métodos de interpretação resultou numa taxa de concordância baixa (71,7\%; kappa $=0,41)$. A sensibilidade da análise computadorizada para os parâmetros indicativos de resultado perinatal desfavorável foi superior à da análise visual, porém essa diferença não mostrou significância estatística $(p>0,05)$. Por outro lado, a análise visual apresentou especificidade para os mesmos parâmetros, analisados individualmente ou em conjunto, maior que a análise computadorizada $(\mathrm{p}<0,001)$. Os resultados falso-negativos em relação à acidose neonatal, tanto da análise visual quanto da computadorizada, corresponderam, em sua maioria, a intercorrências nos partos, não previsiveis por este método propedêutico. Concluiu-se que a interpretação tradicional da cardiotocografia anteparto não foi superada pela análise computadorizada e que deve-se continuar a utilizá-la para a avaliação do bem-estar fetal em gestações de alto risco.

Palavra-chaves: Monitorização fetal. Cardiotocografia. Anóxia perinatal.

\section{Efeitos sobre o Endométrio e o Padrão de Sangramento da Adição Seqüencial Cíclica do Acetato de Ciproterona à Terapêutica de Reposição Estrogênica Continua em Pacientes Pós-Menopáusicas}

Autor: Alberto Soares Pereira Filho

Orientador: Prof. Dr. César Eduardo Fernandes

Tese apresentada à Faculdade de Ciências Médicas da Santa Casa de São Paulo para obtenção do Título de Doutor em Medicina, na área de Tocoginecologia em 9/12/99.

Foram estudadas 41 mulheres pós-menopáusicas, tratadas durante 12 meses com a associação de $2 \mathrm{mg} /$ dia de valerato de $17 \mathrm{~b}$-estradiol em ciclos de 28 dias e acetato de ciproterona ( $1 \mathrm{mg} /$ dia do $19^{\circ}$ ao $28^{\circ}$ dia), em regime combinado seqüencial sem pausa, com a finalidade de avaliar os efeitos sobre o endométrio e o padrão de sangramento. Para estudo histológico endometrial, as pacientes foram submetidas a duas biópsias endocavitárias uterinas, a primeira antes do início e a segunda ao final dos 12 meses de tratamento. Foram realizadas consultas de avaliação aos três, seis, nove e 12 meses, quando eram registrados os padrões de sangramento. O padrão amenorréico inicial manteve-se em 22 pacientes $(53,7 \%)$ no terceiro mês e em $20(48,7 \%)$ no final do tratamento. O padrão de sangramento regular que se observou no terceiro mês de tratamento em 17 pacientes $(41,4 \%)$ tendeu a se manter ao longo de todo o período observado, aparecendo em 19 casos $(46,6 \%)$ no $12^{\circ}$ mês de tratamento. Apenas duas pacientes (4,9\%) apresentaram sangramento irregular no terceiro mês e ao final do estudo. No início do estudo, 33 pacientes $(80,4 \%)$ apresentavam endométrio atrófico e oito pacientes $(19,6 \%)$ exibiam endométrio proliferativo. Não se conseguiu de- 